\title{
Analysis of Conducting and Insulating Surfaces by Means of Secondary Ion Mass Spectrometry (SIMS)
}

\author{
M. De Paz and C. Macciò \\ Istituto di Scienze Fisiche dell'Università di Genova \\ and Gruppo Nazionale di Struttura della Materia del CNR, unità di Genova, Italy
}

(Z. Naturforsch. 30 a, 831-834 [1975] ; received April 28, 1975)

\begin{abstract}
Using a simple home built apparatus, the surface contamination of various samples maintained at high vacuum conditions is investigated by means of SIMS technique.

On a polycrystalline copper surface, at a sample temperature of about $140 \mathrm{~K}$, residual water vapour condensation occurs and ions $\mathrm{H}^{+}\left(\mathrm{H}_{2} \mathrm{O}\right)_{n}$ with $1 \leqq n \leqq 11$ appear in the positive ion spectrum.

On the contrary, on a LiF crystal no water contamination is observed down to $120 \mathrm{~K}$; moreover, on a KI sample, one of the most hygroscopic salts among the alkali halides, only at $130 \mathrm{~K}$ an appreciable $\mathrm{H}_{2} \mathrm{O}$ contamination is detected.
\end{abstract}

\section{Introduction}

Among the various processes occurring during the sputtering of a solid surface bombarded with an energetic ion beam, the emission of secondary ions from the upper layers of the solid is observed. The mass analysis of these ions gives rise to the so called "Secondary Ion Mass Spectrometry" (SIMS). For a long time this technique has been used to investigate the bulk composition of the solid, since the high primary ion beam intensities generally used were sputtering away a single monolayer in less than one second. Recently, however, Benninghoven has introduced the static method of SIMS ${ }^{1}$, which is apt to investigate the first monolayer without appreciably disturbing the surface composition. In fact the low primary ion current density employed $\left(10^{-9} \mathrm{~A} / \mathrm{cm}^{2}\right)$ sputters one monolayer in about $10^{4} \mathrm{sec}$. Such a method may be applied to study various problems ranging from adsorption and surface catalysis ${ }^{2,3}$ to the qualitative (and hopefully quantitative) analysis of substances present as impurities on the surface. This last application is inter- esting to our laboratory, where crystal surfaces are investigated by means of molecular beam scattering experiments ${ }^{4}$. It is obvious that the knowledge of surface composition is vital to a correct interpretation of the observed diffraction patterns.

In this paper we describe the preliminary results obtained with a static SIMS apparatus built to analyze the surface of conducting and insulating materials. The results are so encouraging that the apparatus is now being duplicated in a more sophisticated version as a part of a rotating cryostat where also molecular beam scattering experiments on various surfaces are to be performed.

\section{Apparatus}

A perspective view of the apparatus is shown in Figure 1. The primary ion source $S_{1}$ and the electron source $S_{2}$ are home built following the sketches of Figure 2. Both gave excellent performances provided that emission currents were regulated with precision feedback electronic units. Typical data for

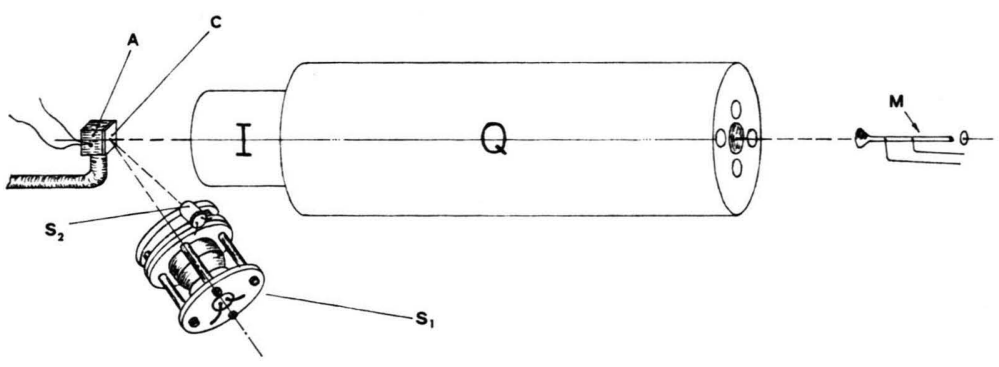

Fig. 1. Experimental set up. $\mathrm{C}=$ sample; $\mathrm{A}=$ sample holder; $\mathrm{S}_{1}=$ primary ion source; $\mathrm{S}_{2}=$ elec tron source; $\mathrm{Q}=\mathrm{R} . \mathrm{F}$. quadrupole mass filter; $\mathrm{I}=$ ionizer; $\mathrm{M}=$ electron multiplier.

Reprint requests to Dr. M. De Paz, Istituto di Scienze Fisiche dell'Università di Genova, Viale Benedetto XV genova, Italy. 


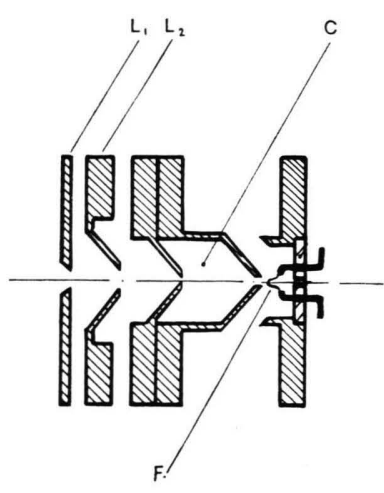

primary ion source $S_{1}$

Fig. 2. $\mathrm{S}_{1}$ and $\mathrm{S}_{2}$ sections. $\mathrm{F}=$ filament; $\mathrm{C}=$ ionization chamber; $\mathrm{B}=\mathrm{Ni}-\mathrm{Cr}$ box; $\mathrm{L}_{1}=$ extractor; $\mathrm{L}_{2}=$ collimator.

$\mathrm{S}_{1}$, as a static SIMS source, are:

$$
\begin{array}{ll}
\text { electron energy } & =200 \mathrm{eV} \\
\mathrm{Ar}^{+} \text {ion energy } & =1000 \div 2500 \mathrm{eV} \\
\text { ion current density } & =10^{-9} \mathrm{~A} / \mathrm{cm}^{2} \\
\text { source pressure } & =10^{-6} \text { torr. }
\end{array}
$$

As a surface cleaning device the ion current density was raised to about $5 \cdot 10^{-7} \mathrm{~A} / \mathrm{cm}^{2}$ by increasing the Ar pressure up to about $10^{-4}$ torr in the ionization chamber.

The electron source $\mathrm{S}_{2}$ is operated only when SIMS spectra are performed on insulating surfaces, in order to avoid space charge effects on the sample. A broad electron beam is produced with an energy low enough $(5 \div 7 \mathrm{eV})$ to avoid spurious ionization processes; the beam intensity is regulated to provide the maximum SIMS ion yields.

The primary ion beam impinges on the surface $\mathrm{C}$ at an angle of $55^{\circ}$ while the R.F. quadrupole Q is situated with its axis normal to the surface. This arrangement has been chosen to optimize the requirements of a good SIMS analysis i. e.:

a) maximum secondary ion yield, which, according to Kaminsky ${ }^{5}$, is obtained for an incidence angle ranging between $45^{\circ}$ and $70^{\circ}$.

b) maximum efficiency of collection of sputtered ions which are roughly distributed according to a cosine law.

c) minimum detection of elastically scattered primary ions.

The sample $\mathrm{C}$ is supported by the copper block A which can be cooled or heated in the temperature range $80 \div 600 \mathrm{~K}$, measured with an $\mathrm{Au}-\mathrm{Au}, \mathrm{Fe}$ thermocouple. Insulating crystals are mechanically clamped on the block. Conducting samples are to be thermally anchored to A but electrically insulated from ground since the surface potential must be regulated to optimize the detected ion intensity.

The R.F. quadrupole mass filter (from Extranuclear) is followed by the electron multiplier $\mathbf{M}$ (a spiraltron from Bendix) which has a gain of about $10^{7}$, so that ion currents as low as $10^{-17} \mathrm{~A}$ can be detected.

The SIMS elements are situated in a vacuum chamber maintained at an ultimate pressure of $2 \cdot 10^{-7}$ torr by an oil diffusion vacuum pump coupled with a liquid nitrogen trap. During the analysis the pressure rises to about $8 \cdot 10^{-7}$ torr when $\mathrm{Ar}$ gas is fed into the primary source $\mathrm{S}_{1}$. The ionizer I of the quadrupole can be used to analyze the residual gas in the chamber. During a SIMS analysis, it is used as an ionic lens set to optimize the output signal.

\section{Results and Discussion}

The first surface examined with our apparatus was a plate of polycrystalline copper mechanically polished. The spectrum obtained didn't show the presence of copper but only peaks from heavy hydrocarbons (Figure 3).

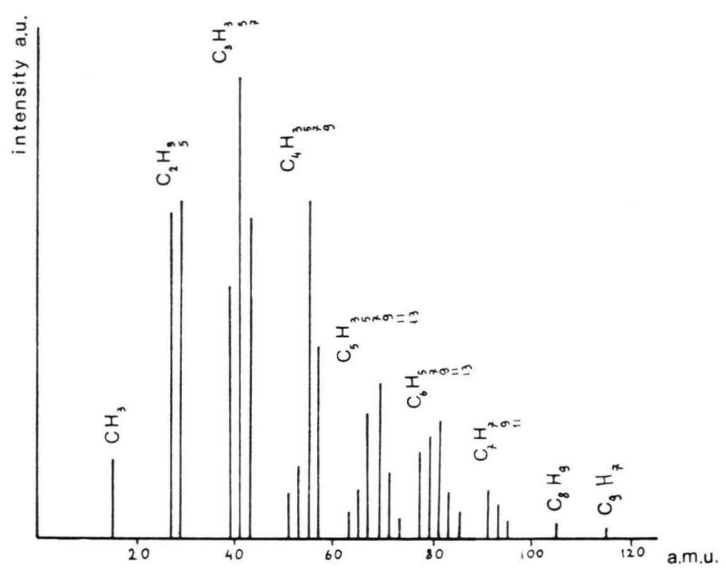

Fig. 3. A spectrum showing a heavy hydrocarbon contamination on the copper sample; it is characterized by a series of fragment peaks at odd masses.

The surface was then thoroughly scrubbed with a clean steel blade; successively it was heated up to $250^{\circ}$ in vacuo and repeatedly bombarded with an ionic current density of $10^{-7} \mathrm{~A} / \mathrm{cm}^{2}$. 
After these treatments we finally observed the two isotopes of $\mathrm{Cu}$ at 63 a.m.u. and 65 a.m.u. in the natural ratio of about $7 / 3$. However, the most intense peaks were due to $\mathrm{Na}^{+}$(at 23 a.m.u.) and $\mathrm{K}^{+}$(at 39 and 41 a.m.u.) coming from impurities of the metal surface. In fact the alkali metals have a very low ionization potential which favours their emission in ionic form by a factor of $10^{3}$ with respect to other metals.

When the sample temperature is lowered, condensation of residual gases occurs, so that the dominant spectrum, down to $-120^{\circ} \mathrm{C}$, is that of hydrocarbons coming from oil vapours of the diffusion pump.

At $-130{ }^{\circ} \mathrm{C}$ the appearance of $\mathrm{H}_{3} \mathrm{O}^{+}$ions indicates a sudden condensation of water vapour. A semilog plot of $\mathrm{H}_{3} \mathrm{O}^{+}$ion intensity versus $\mathrm{l} / T$ (see Fig. 4) is interpreted as an activated process with an energy of $13 \pm 2 \mathrm{kcal} / \mathrm{mole}$, very close to the

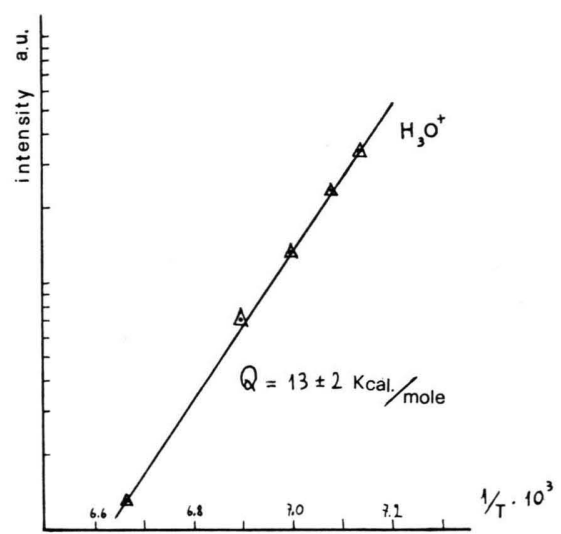

Fig. 4. A semilog plot of $\mathrm{H}_{3} \mathrm{O}^{+}$intensity versus $1 / T$, where $T$ is the sample temperature; from it we can deduce that $I \propto e^{Q / R T}$ where $Q=13 \pm 2 \mathrm{kcal} / \mathrm{mole}$.

sublimation heat of ice. Simultaneously complex ions, such as $\mathrm{H}_{3} \mathrm{O}^{+}\left(\mathrm{H}_{2} \mathrm{O}\right)_{n}$ with $1 \leqq n \leqq 10$, are formed. These species have been previously observed in mass spectra of water vapour at relatively high pressure ${ }^{6}$ and their ice structure calculated with a C.N.D.O. molecular orbital technique ${ }^{7}$. Present experiments confirm that these ions can be formed directly from a solid surface, which has presumably an ice like structure.

Measurements were then performed on insulating materials using the electron source $S_{2}$ as described in the previous section. Our main purpose was to examine the surface impurities of alkali halide samples, since in our laboratory scattering experi- ments of $\mathrm{He}$ atoms on $\mathrm{LiF}$ crystals have been previously performed and the possible presence of orderly adsorbed water molecules on the surface has been surmised ${ }^{4}$. The LiF sample showed no water contamination even at temperatures as low as $120 \mathrm{~K}$. Moreover, hydrocarbons impurities were apparently less easily adsorbed on this surface than on that of copper.

The room temperature spectrum of $\mathrm{LiF}$ after treatment at $600 \mathrm{~K}$ (as in the scattering experiments) shows practically only peaks due to $\mathrm{Li}^{+}, \mathrm{Li}_{2}{ }^{+}$ and $\mathrm{Li}_{2} \mathrm{~F}^{+}$(Figure 5). Similar results have been recently obtained by Hoinkes, Nahr and Wilsch ${ }^{8}$.

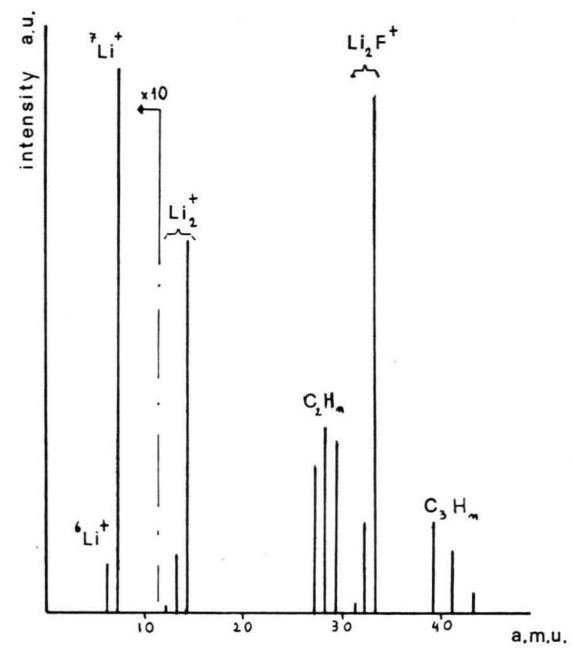

Fig. 5. A spectrum obtained at room temperature on the $\mathrm{LiF}$ sample surface after treatment at $600 \mathrm{~K}$.

The adsorption of water on alkali halide surfaces has been further investigated by the SIMS analysis of the hygroscopic salt KI. On a freshly cleaved sample, an appreciable water contamination is observed only below $130 \mathrm{~K}$ and this disappears completely when the temperature is raised by a few degrees.

At high temperature the spectrum shows only $\mathrm{K}^{+}$, $\mathrm{K}_{2}^{+}, \mathrm{KI}^{+}$and also $\mathrm{I}^{+}$. Other trace peaks, probably due to lattice impurities, such as $\mathrm{Na}^{+}$and $\mathrm{K}_{2} \mathrm{Cl}^{+}$, are also observed.

In conclusion, our preliminary studies of conducting and insulating surfaces by Secondary Ion Mass Spectrometry have confirmed that this technique is a powerful tool to investigate the surface contamination of samples; this is essential to a correct interpretation of surface properties. 
1 A. Benninghoven, Surface Science 35, 427 [1973].

2 M. Cavallini and G. Nencini, 9th International Symposium on Rarefied Gas Dynamics, Göttingen, July 15-20, 1974

3 M. Cavallini and G. Nencini, 5th Symposium International sur les Jets Moleculaire, Nice, April 7-12, 1975.

4 G. Boato, P. Cantini, U. Garibaldi, A. C. Levi, L. Mattera, R. Spadacini, and G. E. Tommei, J. Phis. C: Solid State Phys. 6, L394 [1973].
5 M. Kaminsky, Atomic and Ionic Impact Phenomena on Metal Surfaces, Academic Press, New York 1965.

6 M. De Paz, J. J. Leventhal, and L. Friedman, J. Chem. Phys. 49, 5543 [1968].

7 M. De Paz, S. Ehrenson, and L. Friedman, J. Chem. Phys. 52, 3362 [1970].

$8 \mathrm{Nahr}$, private communication. 